\title{
METHODOLOGY
}

\section{Strategy for prevention and control of the risks due to noise}

\author{
J Malchaire
}

\begin{abstract}
Objectives-To propose a strategy for progressively controlling the exposure to noise in industry as much as possible. To propose a method that could, in the first stage, be used by the workers and management themselves to control exposures to noise as much as possible, and then, in later stages, when necessary, progressively call in the assistance of specialists and experts to identify more complex solutions and organise personal protection and medical surveillance.

Methods-The strategy includes three stages. Stage 1 is observation, simple and easy to use by the workers to recognise the problems, identify straightforward solutions, and call for assistance when needed. Stage 2 is analysis, more complex but more costly, performed with the assistance of occupational health specialists to identify more technical control measures and set up a programme to conserve hearing. Stage 3 is expertise, performed with the assistance of acoustic experts for special measurements and control measures.

Conclusions-The proposed strategy enriches the assessment procedure that is usually recommended, by providing for one preliminary stage used by the people directly concerned. It explicitly recognises (a) the competence of the workers and management about their working conditions and (b) that knowledge and measurements of acoustics are not an absolute prerequisite for solving-at least partly-noise problems. It attempts to organise in sequence and optimise the cooperation between the workers, the occupational health specialists, and the experts in acoustics.

(Occup Environ Med 2000;57:361-369)
\end{abstract}

de Louvain, Unité

Hygiène et Physiologie du Travail, Clos

Chapelle-aux-Champs

30-38, B - 1200

Bruxelles, Belgium

Correspondence to: Professor J Malchaire malchaire@

HYTR.UCL.AC.BE

Accepted 26 January 2000 Keywords: risk assessment; small and medium sized enterprises; hearing; hearing conservation programmes

Many books, papers, and documents describe the criteria for programmes to conserve hearing. ${ }^{1-7}$ Surprisingly, few give a clear definition of the objectives of such programmes, other than to prevent employees from develop- ing noise induced hearing loss at work. ${ }^{8}$ On the contrary, they usually define their components, which according to Suter and Franks ${ }^{9}$ are $(a)$ monitoring exposure to noise, $(b)$ engineering and administrative controls, (c) audiometric evaluation, (d) use of devices to protect hearing, (e) education and motivation, $(f)$ record keeping, and $(g)$ evaluating the programme. Their document, 70 pages long, describes in detail these seven components.

Leinster et $a l^{10}$ investigated the managerial, organisational, and psychological factors which are involved in the limitation of hearing loss induced by noise in the workplace, to improve the understanding of why people in industry fail to take action to avoid hearing loss induced by noise. Surveying 48 organisations, they showed that only $40 \%$ had carried out assessments which were adequate to comply with the legislation, most had not developed the engineering control measures as far as they might, $50 \%$ had instructed the workers on how to use personal protection, and $26 \%$ had a specific noise training programme.

These conclusions are disappointing. The study was conducted in companies with $\geqslant 150$ employees. As about $50 \%$ of the workforce is employed in smaller companies, it might be suspected that compliance with the noise at work regulations and therefore their efficiency is even worse than it seems.

The reasons are many. Leinster et $a l^{10}$ identified several of them: noise at work taken for granted and not perceived as a serious problem, lack of leadership, no clear allocation of operational responsibilities, lack of technical competence, presumption that control measures are expensive. Royster and Royster ${ }^{8}$ mentioned the same reasons, with an additional one: overreliance on contractors to provide hearing conservation programme services.

These conclusions are not specific to hearing conservation programmes and are most likely valid for any health and safety problem.

They put the pressure on employers, stress- ing the necessity for them to be motivated and to motivate the employees.

I suggest that the scientific community might be partly responsible for this, as it has failed to provide simple, inexpensive, efficient methods for employers and the industry in general to prevent or control noise problems, so that they 
could themselves recognise the problems and start doing something.

In a recent encyclopaedia, ${ }^{11}$ some guidelines for the development of a programme to conserve hearing are proposed. Under the first item-sound exposure surveys - they indicate that representative individual exposures to daily noise (have to be) established for all job classifications exposed to noise, and that a noise map of the plant (must be) posted. Other documents ${ }^{9}$ recommend similar early stages.

The question is whether or not employers are aware and can use concepts such as representativeness, daily exposures, job classifications, and noise maps. One remarkable example of an unused (and probably unusable) assessment method was published by myself. ${ }^{12}$ It can be argued that to make people aware and competent is the realm of occupational health specialists and in particular of industrial hygienists. If, indeed, such specialists are required to assess and improve the noise conditions, this implies that hearing conservation programmes can only be developed when such specialists are available or called in. This is probably the case in large companies. It is less likely in small and medium sized enterprises and $50 \%$ of the work force will probably miss such programmes.

A different approach is to develop and propose a simple method that can be understood and used by all companies whatever their size and their health and safety management so that they at least become aware of the problem, and can come up with some straightforward solutions. Occupational health specialists would be called in at later stages of the procedure if and when needed, to assist in finding more complex solutions and organise personal protection and medical surveillance programmes.

Here I describe a strategy in three stages. Its purpose is to make it possible to initiate and conduct a policy of prevention in any company of any size or type, based on the expertise available inside and outside this company.

A similar method was proposed for the prevention of heat exposure. ${ }^{13}$

\section{Philosophy of the method}

The primary objective of an analysis of the working conditions should not be simply to assess the risks, but to prevent, to eliminate, or at least to reduce these risks. The method described in this document is therefore not to measure the risk but to collect the information progressively, as it is needed for the research of prevention or control measures.
The key postulates of the strategy are that measurements in themselves do not solve a problem and that the solution of a problem does not necessarily require measurements.

The number of working situations with exposure to noise is very high and it would be a Utopia and totally unrealistic to require that all of them be studied in detail. This would be useless, as, in most cases, at least partial solutions can be found easily, based on simple and straightforward observations. In some cases, however, a more detailed analysis is necessary, including measurements, and in some particular cases, expertise can be required, based on more complex evaluation techniques.

The method is therefore based on a progressive approach in three stages with the characteristics summarised in table 1 .

The first stage, observation, should be usable by people from the company who have full knowledge of the working conditions, but do not necessarily have training in acoustics or audiology. Its aim is to characterise the working situation in all work circumstances, not at a specific time, and to determine what can be done immediately to reduce or eliminate these problems. Clearly this must be done by or with the help of the workers themselves, who know best the working conditions. Competence in acoustics should not be required at this stage, although it would of course be useful.

At the end of this stage, the users should decide whether the problem is satisfactorily controlled or if the assistance of a specialist is needed.

The second stage, analysis, should be conducted by the same people, but with the assistance of specialists who have specific training in conserving hearing. It deals with the working situation under particular circumstances (a specific operation or machine, etc) identified during the first stage and might require common measurements.

Again, the method must be designed to find technical solutions. When exposure is unacceptable, organisational measures, personal protection, and medical surveillance must be implemented.

At the end of this second stage, most of the conditions should be under control. It might be, however, that in very special cases, due to unusual circumstances, an unacceptable risk of discomfort or hearing impairment remains.

The third stage, expertise, should then be used, again, by the same people, but with the additional assistance of highly specialised experts. It will deal with complex circumstances and might require complex or special

Table 1 Characteristics of the three stages of the prevention strategy

\begin{tabular}{llll}
\hline & Stage 1 Observation & Stage 2 Analysis & Stage 3 Expertise \\
\hline When? & All cases & More complicated cases & Very complex cases \\
How? & Qualitative observations & Ordinary measurements & Specialised measurements \\
Cost? & Low & Average & High \\
By whom? & People from the company & Same & Same \\
& & + Specialists & + Specialists \\
& & & + Experts \\
Competence: & High & Average & Low \\
Work situation & Average & High & Specialised \\
Ergonomics & & & \\
\hline
\end{tabular}


measurements. This type of investigation will usually be more expensive and therefore must be limited to conditions where it is essential.

\section{Criteria for observation, analysis, and expertise}

A similar strategy was developed for thermal problems at work.

A survey was conducted on some 40 people with different backgrounds, in different types of industries, and in different countries. They were asked what time, efforts, and competence were available in industry to deal with the thermal problems, and what criteria should fulfil a prevention strategy such as the present one to be of interest to them in practice. The results are not specific for heat problems and will be summarised here.

To be useful to the workers and their management, the first stage must avoid any specialised terms, must be straightforward, and require $<90$ minutes to be used in a given workshop. It must be explicitly oriented towards prevention and control of the problems, and avoid the collection of any unnecessary data. Suggestions must be made about the prevention measures.

At the second stage, the method can be more complex and involve measurements. However, non-essential concepts and measurements must be avoided, as the occupational specialists (occupational physicians, occupational hygienists, ergonomists) still have limited training in acoustics and measuring equipment remains expensive and uncommon. These measurements should be oriented again towards the search for technical solutions rather than towards risk assessment. Such an analysis should take less than 1 day to be convenient to use under a broad range of conditions.

The strategy should define how to organise the cooperation of the different people in order, from the workers to the experts, defining who should do what, when one person calls in the others, and how they complement each other.

The strategy proposed in the appendix attempts to use these criteria.

\section{Description and justification of the strategy}

For each stage, the document recalls briefly the objectives and who should be the users. Then the procedure is defined.

The document should be kept short and concise, guiding the users in their approach to working conditions, and providing only the information they need at that time, at the workplace, to estimate and conclude.

The document reproduced in the appendix is normally accompanied by a short guide that provides some additional information at each stage. This document is not discussed here.

\section{STAGE 1, OBSERVATION}

Contrary to common beliefs, noise ordinances - or at least the European noise Directive $^{14}$ - do not recommend that the personal level of exposure to noise needs to be $<90$ or $85 \mathrm{~dB}(\mathrm{~A})$, but that "the risks resulting from exposure to noise must be reduced to the low- est level reasonably practicable, taking account of technical progress and the availability of measures to control the noise, in particular at source" (Article 5.1). This means that a personal noise exposure level of-for instance- $-98 \mathrm{~dB}(\mathrm{~A})$ must be reduced to 75 $\mathrm{dB}(\mathrm{A})$ if reasonably possible, and to $94 \mathrm{~dB}(\mathrm{~A})$ if only a $4 \mathrm{~dB}$ reduction is possible; in which case, the exposure must be documented and additional measures must be taken (personal protection, audiometry, workers' information).

This implies therefore, that instead of measuring noise, it should first be determined whether anything can be done to improve the sources, the tasks, the tools, or the environment, whatever the noise levels. Measurements might be needed at a later stage to deepen the analysis. This also corresponds to the requirements of the European Directive ${ }^{14}$ that noise "shall be assessed and, when necessary, measured ..." (Article 3.1).

Also, any measurement raises the problem of representativeness. Although textbooks in acoustics as well as the European Directive (in its annex 1) provide detailed information about the instrumentation, the location of the microphone (10 cm from the head !), they overlook or deal only briefly with the problem of representativeness. As our previous paper ${ }^{12}$ clearly showed, the accurate evaluation of the level of personal exposure to noise is extremely complex in most industrial environments and evaluation should only be attempted when it is necessary - that is, when all ways of solving the noise problem simply have been tried.

It could be argued, that even for assessing what can be done to lower a given noise, measurements would help. Our experience, however, is that measuring instruments are often misused (too close or too far from the source, without recognising other interfering sources, etc) or the results misinterpreted (ignoring the reverberant field). Therefore, we propose that measurements by untrained people might be misleading and must be avoided.

As stage 1, observation, is designed to be used by people in the field without necessarily the assistance of specialists, it deliberately discourages measurements and relies on the opinions of these people directly concerned.

Obviously, these opinions and voice levels are not accurate. However, contrary to point measurements, they have at least the advantage of reflecting how these workers "live" their noise exposure, not only at a given time but in the different work circumstances.

Stage 1 concentrates therefore on the identification of the noise sources. It starts by asking for a drawing of the working area to be made, with the exact location of the sources of noise and the workers. Then, for each workplace, it relies on the voice level that has to be exerted to be understood at a distance of $0.5 \mathrm{~m}$, to assess the severity of the problem. This was chosen as it avoids the problem of measurements and corresponds to problems people might have encountered repetitively in their daily lives.

From this voice level, a rough estimation of the noise level is deducted. This is based on the International Standards Organisation (ISO) 
9921 standard $^{15}$ defining the communication distances as a function of the speech interference level. No mention is made of this standard in the document, as it is not readily available, difficult to understand, and suggests octave band analysis of the noise level.

From this, the severity of this particular situation is assessed assuming that the noise was continuous, and ranged from light discomfort to high risk of hearing impairment. The scale used is based on common knowledge for "light" and "high" discomfort and on the ISO 1999 standard $^{16}$ for the risk of hearing impairment. Indeed, from this standard, it can be derived that the percentage of additional subjects who, at age 55 and after 35 years of exposure, will develop a mean hearing impairment (mean at $1 \mathrm{kHz}, 2 \mathrm{kHz}$, and $3 \mathrm{kHz}$ ) $>25$ $\mathrm{dB}$, is $3 \%$ for a personal noise exposure level equal to $85 \mathrm{~dB}(\mathrm{~A}), 10 \%$ for 90 , and $49 \%$ for $100 \mathrm{~dB}(\mathrm{~A})$. This hearing impairment is defined as a "material hearing impairment" by the National Institute for Occupational Safety and Health (NIOSH). ${ }^{17}$

A scale with five points was deliberately chosen to show different degrees of severity and avoid the common misbelief that a situation is unacceptable $>85 \mathrm{~dB}(\mathrm{~A})$ (or 90) and acceptable below this level.

The users are then invited to study each source carefully and look for straightforward control measures. As these users are the workers themselves and their technical management, it can be expected that they know their equipment in detail and can, more easily than a specialist, identify efficient control measures that will not interfere with the tasks. They do know in general what types of gears are used, what parts vibrate, what can be enclosed, etc.

The document suggests a series of possible actions but mainly draws attention to the different aspects to be considered. It also mentions solutions that cannot be implemented without the assistance of specialists: equilibration of parts, duct silencers, absorbing materials, etc. These were included deliberately to help these users to recognise when they need this assistance and to call in these specialists with specific objectives.

While discussing possible solutions, the users are invited to consider their potential efficiency in controlling the noise. It could be argued that the workers and their management will most likely not have the knowledge needed for these predictions to be reliable. Although this is likely, it is also true that in many occupational health studies, even performed by specialists, much more time and effort is spent on diagnosis than on prevention, and recommendations are sometime made without even attempting to evaluate how much the situation will really be improved. Bringing attention to this efficiency is therefore a step forward, as long as the users recognise the uncertainty of their estimations.

The procedure ends up by inviting the users to decide who will do what and when and deciding whether a specialist must be called in to help perform a more detailed analysis.
STAGE 2, ANALYSIS

Stage 2, analysis follows roughly the same procedure, this time going into detail in the particular situations that were identified as unsatisfactory at the end of stage 1, the observation.

Firstly, an attempt is made to assess the level of the workers' personal exposure to noise. As the analysis will be managed by trained occupational health specialists, concepts such as homogeneous exposure groups and representative samples may be used. They are invited to decide the appropriate time and duration of the measurements, to control the validity of the measuring technique, to perform the measurements, and to estimate the duration of exposure at each measured level, if different conditions exist.

The measurements are restricted to A weighted noise levels. They should preferably be performed with a calibrated exposimeter recording, for instance, the equivalent noise level every 15 seconds. Such instruments are continuously becoming more available and cheaper. Still, they are at the disposal of few specialists and are little used. We chose therefore not to specify the measuring technique, leaving the specialists to do what they are able to do, in the best conditions.

This is even more true for frequency analyses, which would obviously provide useful information to optimise some control measures - such as absorbing or damping materials - therefore we chose once again not to include these costly measurements in the analysis, hoping that the specialists who have the means and the training to make such measurements will know how they can enrich the standard procedure.

From the equivalent noise levels $\mathrm{L}_{\text {Aeq }}$ and durations of exposure, it is recommended to compute partial personal noise exposure levels $\mathrm{L}_{\mathrm{PE}, 1}$. To our knowledge, this concept is new. It is actually the personal noise exposure level if all other noise exposures were insignificant. These partial $\mathrm{L}_{\mathrm{PE}, 1}$ levels make it possible to determine what work sequences, what situations, or what noise sources are responsible for most of the risk of discomfort or hearing impairment.

The reduction factor to derive $\mathrm{L}_{\mathrm{PE}, 1}$ from $\mathrm{L}_{\mathrm{Aeq}} \mathrm{i}$ is given by a formula as well as in a tabular form. Graphs were extensively used in the past when calculation possibilities were limited. They should now be considered obsolete.

The total personal noise exposure level $\left(\mathrm{L}_{\mathrm{PE}}\right)$ is estimated in the usual way by the addition of the partial exposure levels.

The scale of interpretation can be more quantitative than in stage 1, observation. Again, the ISO $9921^{15}$ and the ISO $1999^{16}$ standards are used respectively to scale the discomfort and the risk of hearing impairment, and the interpretation is not done simply in terms of compliance or not with a limit, but on a rather continuous scale, from light discomfort, to a probability that $75 \%$ of the exposed group has a "material hearing impairment" at the age of 55 years in case of an exposure to these conditions during 35 years. 
Discomfort is linked not to the level of personal exposure to noise but rather to the equivalent level in the short term.

The risk of hearing impairment on the contrary is related to the $\mathrm{L}_{\mathrm{PE}}$. A double scale for hearing impairments of 25 and $50 \mathrm{~dB}$ is used to avoid the misconception that a worker is "deaf" above a given hearing impairment and is "not deaf" below it.

The users are then invited to go through the list of possible solutions and identify what could be done to reduce the risk of discomfort or hearing impairment.

As in stage 1 , observation, they are invited to estimate what the situation might be if these solutions were implemented and to estimate the residual risk. If this risk is unacceptable, additional efforts and information are required: a stage 3 , expertise, must be undertaken with the further assistance of an expert.

They will finally draw up the inventory of the technical measures, define deadlines, and allocate responsibilities for actions.

At this stage, more than in stage 1, observation, the users have adequate training and enough information to determine whether personal protection must be worn; what protection should be carried out, by whom, when and for how long; who should participate in the audiometric programme, and when.

The document draws attention to some main aspects of personal protection. These recommendations are based on the fact that the best protective device is the one that is worn for the longest period of time. ${ }^{17}{ }^{18}$ Emphasis is therefore placed on convenience of use, comfort, and aesthetics, rather than on the intrinsic attenuation capacity.

STAGE 3, EXPERTISE

This section is not developed in the appendix. Indeed, at this stage, the overall situation should be known and attention will concentrate on very specific items such as the reverberation of the hall, the damping of a vibrating structure, the silencing of a resonating structure, etc. This will involve specific, highly complex measurements and the experts should know what to do in a given case.

The only aspect to stress is the absolute necessity to assist this expert. Much too often, the problem is transferred to the expert and he is expected to solve it by himself.

As underlined in table 1, this strategy is based on the expertise of the workers complementing the expertise of the specialists.

\section{Conclusion}

The strategy rests on three basic principles.

IT IS REALISTIC

Noise regulation has existed for decades. Still, many workers are exposed to harmful noise. Motivation is limited in industry, as time, money, technical resources, and competence in acoustics are limited. The assessment and prevention procedures must be optimised, starting not from the ideal procedure as scientists would see it, but from what industry is willing and able to do.

\section{IT IS PARTICIPATIVE}

The workers and their management play the essential part in the dynamics of the improvement of the working conditions. Occupational health specialists and experts are there to assist, to identify the best technical and organisational control measures.

The strategy is structured in three stages that require complementary knowledge and competence.

At the first stage, observation, involves knowledge of the industrial process, the machines, and the working procedures.

At the analysis stage the specialists assist with education and training about the general methodological aspects, the common measuring and evaluation techniques, and the main technical solutions.

At the expertise stage, when it is absolutely necessary, assistance is sought from highly trained experts who will bring their specific knowledge to identify particular solutions.

The strategy is not designed for experts but for the industry, calling in experts when needed. This itself might be Utopian in many industrial settings, at least at the present time. Specialists can still use the strategy directly to initiate the procedure and show its simplicity and usability. Contrary to what is often done, the experts are not called in to be responsible for finding the solutions, but the whole process of searching for solutions is carried out in full partnership.

The strategy does not therefore exclude the participation of a specialist from the start. Simply, it does not rely on this.

It is hoped that this approach will make possible, at least, some improvement of the working conditions in some small and medium sized enterprises.

\section{Appendix: strategy for prevention and control of the risk due to noise \\ STAGE 1, OBSERVATION \\ Objectives \\ The objectives are:}

- To collect general information on the noise sources and conditions of exposure to noise in the field

- To find technical measures that can immediately be used to eliminate or control the risk

- To find whether a detailed analysis (stage 2) is necessary, with what priority, and with what objectives.

Table 2 Magnitude of noise and risk of hearing loss at the end of the day, in the opinion of the workers

\begin{tabular}{llllll}
\hline Voice level & Normal & Loud & Very loud & Shouting & $\begin{array}{l}\text { Maximum } \\
\text { shouting }\end{array}$ \\
\hline Level $(\mathrm{dB}(\mathrm{A}))$ & 50 & 70 & 85 & 90 & 100 \\
Interpretation & Light discomfort & High discomfort & Low risk & Medium risk of hearing loss & High risk \\
\hline
\end{tabular}


Table 3 Worker's report

\begin{tabular}{llllllll}
\hline & Before & \multicolumn{9}{l}{ After } \\
\cline { 2 - 3 } Workplace & $\begin{array}{l}\text { Voice } \\
\text { level }\end{array}$ & $\begin{array}{l}\text { Noise } \\
\text { level }\end{array}$ & Risk & & $\begin{array}{l}\text { Voice } \\
\text { level }\end{array}$ & $\begin{array}{l}\text { Noise } \\
\text { level }\end{array}$ Risk & $\begin{array}{l}\text { Need for an } \\
\text { analysis }\end{array}$ \\
\hline
\end{tabular}

Who?

People inside the company, the workers themselves and their technical staff, understand the working conditions perfectly.

- The method uses simple vocabulary and words such as "risk", "problem" are used in their common meaning

- The work situation is investigated under all circumstances and not at a given time

- It does not require any measurement.

Procedure

(1) Characterisation of the noise sources

- Indicate the exact location of the workplaces and the workers on a map of the area.

- Identify the main noise sources (examples: compressor, grinder, power saw, etc).

(2) Evaluation of the risk in the present situation at each workplace

- Estimate the voice level spontaneously adopted in this noisy environment

- Derive the order of magnitude of the noise level and the severity of the risk from table 2, and on the basis of the observations already made and of the opinions of the workers (table 2)

- Report the results in the before part of table 3.

(3) Noise control

- Observe each source and look for straightforward solutions to eliminate, reduce, or control the noise emissions: table 4 gives the most common items to consider at this stage

- Report the results as in table 5, indicating who will be responsible, when the modifications are expected to be
Table 5 Actions resulting from table 4

\begin{tabular}{lll}
$\begin{array}{l}\text { Noise } \\
\text { sources or } \\
\text { activities }\end{array}$ & Actions & \\
\cline { 2 - 4 }
\end{tabular}

brought in, and what is the priority of the action.

(4) Evaluation of the residual risk after implementation of the control measures

- Repeat step 2 (evaluation of the risk) after the control measures are taken.

- Complete the after part of table 3 accordingly.

(5) Conclusions

- Is there a need for a detailed analysis (stage 2)?

- For what sources of noise?

- Objective: to reach what level of risk?

- Short term measures (personal protection): what protection, carried out by whom, when, and for how long.

STAGE 2, ANALYSIS

Objectives

For the problems not solved satisfactorily at the previous stage 1 , observation - that is, when the residual risk estimated (table 3 ) at the end of stage 1, observation - is unacceptable.

- Deepen the research for prevention or control measures, with ordinary measurements and more specialised techniques

- Estimate the exposure of the workers

- Search for more complex prevention or control measures

- Organise the hearing conservation programme

- Estimate whether it is necessary to proceed to a more detailed stage 3 , expertise.

Who?

An occupational health specialist trained in hearing conservation can assist the people who performed the stage 1 , observation.

Table 4 Common items to consider and possible solutions

\begin{tabular}{ll}
\hline Items to consider & Possible solutions \\
\hline Vibrations of parts or panels & Tighten parts or panels \\
& Cover them with a rubbery material \\
Install silent blocks & Tilt the plate on which the parts are falling \\
Impacts of parts on a hard surface & Cover it directly or in a sandwich with a rubbery material \\
& Use helicoidal gears \\
Mechanical noise & Use plastic materials \\
& Equilibrate rotating parts \\
& Avoid discontinuities (elbows) or sharp edges in the air stream \\
Aerodynamic noise & Use silencers in ducts \\
& Use exhaust mufflers for decompression air jet \\
Air jets & Use special air guns \\
& Reduce the air velocity of the jet \\
& Avoid the impact of the air jet on a sharp edge or perpendicular to a surface \\
& Use hermetic enclosure covered with rubbery materials \\
Acoustic enclosure on the machine & Install absorbing materials inside \\
Pure tones & Equilibrate rotating parts \\
& Dampen the blade on power saws \\
& Use rubbery materials on resonating parts \\
Relocation of the source & Move the source away from the workers \\
Acoustic treatment of the room & Install a screen between sources and workers \\
& Add some absorbing materials near the source if the room is highly reverberant \\
& Check noise transmission from adjacent rooms or from the outside
\end{tabular}


Table 6 Computation of $k$

\begin{tabular}{|c|c|c|c|c|c|c|c|c|c|c|c|c|c|c|}
\hline Duration/week & $\begin{array}{l}5 \\
\min \end{array}$ & $\begin{array}{l}25 \\
\min \end{array}$ & $\begin{array}{l}50 \\
\text { min }\end{array}$ & $\begin{array}{l}100 \\
\min \end{array}$ & $\begin{array}{l}150 \\
\min \end{array}$ & $\begin{array}{l}225 \\
\min \end{array}$ & $5 \mathrm{~h}$ & $7.5 \mathrm{~h}$ & $10 \mathrm{~h}$ & $15 \mathrm{~h}$ & $20 \mathrm{~h}$ & $25 \mathrm{~h}$ & $30 \mathrm{~h}$ & $40 \mathrm{~h}$ \\
\hline Duration/day & $\begin{array}{l}1 \\
\min \end{array}$ & $\begin{array}{l}5 \\
\min \end{array}$ & $\begin{array}{l}10 \\
\min \end{array}$ & $\begin{array}{l}20 \\
\min \end{array}$ & $\begin{array}{l}30 \\
\min \end{array}$ & $\begin{array}{l}45 \\
\text { min }\end{array}$ & $1 \mathrm{~h}$ & $1.5 \mathrm{~h}$ & $2 \mathrm{~h}$ & $3 \mathrm{~h}$ & $4 \mathrm{~h}$ & $5 \mathrm{~h}$ & $6 \mathrm{~h}$ & $8 \mathrm{~h}$ \\
\hline $\mathrm{k} \mathrm{dB}(\mathrm{A})$ & 27 & 20 & 17 & 14 & 12 & 10 & 9 & 7 & 6 & 4 & 3 & 2 & 1 & 0 \\
\hline
\end{tabular}

- Concepts such as damage, exposure, and risk are used in a more specialist way

- Noise exposure levels are measured, with simple instruments.

Procedure

(1) Exposure of the workers: present situation

- Form groups of workers with the same exposure (homogeneous exposure groups).

- Determine the period (in hours, days, weeks) to cover all exposures to noise (several cycles of work, if they exist)

- Determine the appropriate times and durations for the measurement - During different work phases - At representative dates and hours

- Find the most appropriate measuring techniques

- Measure the equivalent A weighted noise levels $\mathrm{L}_{\mathrm{Aeq}}{ }_{\mathrm{i}}$ for each work phase $\mathrm{i}$

- Evaluate the exposure duration per day or per week for each level of $\mathrm{L}_{\mathrm{Aeq}}{ }_{\mathrm{i}}$

- Compute the partial personal level of exposure to noise $\mathrm{L}_{\mathrm{PE}, \mathrm{i}}$ by subtracting from the $\mathrm{L}_{\mathrm{Aeq}}{ }_{\mathrm{i}}$ the value $\mathrm{k}$ given by the following expressions or table, as a function of the duration of exposure (table 6):

- Compute the personal level of expo-

$$
k=10 \lg \left(\frac{8 \mathrm{~h} \text { or } 480 \mathrm{~min}}{\text { duration per day }}\right)
$$

or

$$
k=10 \lg \left(\frac{40 \mathrm{~h} \text { or } 2400 \mathrm{~min}}{\text { duration per week }}\right)
$$

sure to noise $\mathrm{L}_{\mathrm{PE}}$ by combining successively pairs of $\mathrm{L}_{\mathrm{PE}, \mathrm{i}}$ according to their difference (table 7).

- Estimate the risk in the present situation

- Discomfort: to be assessed by comparing the $\mathrm{L}_{\text {Aeq }} s_{i}$ with the scales in table 8 .

- Hearing impairment: to be assessed by comparing the $\mathrm{L}_{\mathrm{PE}}$ to 85 and 90 $\mathrm{dB}(\mathrm{A})$ and according to the presence or not of noises $>140 \mathrm{~dB}$. Table 9 gives the percentage of subjects who, at the age of 55 years, after 35 years of exposure to the present noise conditions, are expected to have:

- Average hearing loss $>50 \mathrm{~dB}$

\begin{tabular}{|c|c|c|c|c|c|c|c|c|c|c|c|c|}
\hline \multicolumn{13}{|l|}{ Difference in $\mathrm{dB}(\mathrm{A})$ : } \\
\hline Greatest-smallest & 0 & 1 & 2 & 3 & 4 & 5 & 6 & 7 & 8 & 9 & 10 & 12 \\
\hline Total-greatest & 3 & 2.5 & 2.1 & 1.8 & 1.4 & 1.2 & 1.0 & 0.8 & 0.6 & 0.5 & 0.4 & 0.2 \\
\hline
\end{tabular}

Table 7 Author provide table title?
- Average hearing loss $>25 \mathrm{~dB}$

- Prioritisation of the work phases:

- Find the work phases with $\mathrm{L}_{\mathrm{PE}, \mathrm{i}}$ greater than the maximum acceptable level of noise

- Find the main sources of noise responsible for these $\mathrm{L}_{\mathrm{PE}, \mathrm{i}}$

Assessment of the present situation in a particular work situation (table 10).

(2) Detailed analysis of the exposure conditions

- Modification of the work techniques by changing the technology or replacing noisy machines

- Characterisation of the sources of noise

- Identify the causes of the noise (example: vibrations, impact) and the sources of noise (panels and parts to which vibration is transmitted and that radiate the noise)

- Measure the noise level at the sources to identify the real sources of noise and evaluate the directions in which the noise is emitted.

- Reduction of the noise

- Take into consideration the points described in section 3 of stage 1 with particular attention to the transmission of vibration to surfaces or parts.

- Noise propagation

- Take into consideration the points described in section 3 of stage 1 with particular attention to the distances between sources and workers, the directivity of the sources, and the screens between sources and workers

- Acoustic treatment of the room

- Take into consideration the points described in section 3 of stage 1 with particular attention to the echoes (reflection between parallel surfaces), the reverberation time, and any reflecting partitions, ceiling, or ground

- Add absorptive materials if needed.

- Acoustic insulation with the neighbours and with the outside

- Check and improve the tightness of doors and windows

- Eliminate or reduce cracks, openings

Table 8 Levels of voice, discomfort, and $L_{A e q, i}$

\begin{tabular}{lllll}
\hline $\mathrm{L}_{\text {Aeq,i }}(\mathrm{dB}(\mathrm{A}))$ & 50 & 60 & 70 & 80 \\
Voice level & Normal & Raised & Loud & Very loud \\
Discomfort & Low & Medium & High & Extreme \\
\hline
\end{tabular}

Table 9 Expected percentage of workers with hearing loss after 35 years of exposure

\begin{tabular}{lrrrrrrrr}
$\mathrm{L}_{\mathrm{PE}} \mathrm{dB}(\mathrm{A})$ & 85 & 90 & 92 & 94 & 97 & 98 & 99 & 100 \\
$50 \mathrm{~dB}$ & 4 & 5 & 7 & 9 & 15 & 18 & 21 & 26 \\
$25 \mathrm{~dB}$ & 29 & 36 & 40 & 46 & 59 & 65 & 70 & 75 \\
\hline
\end{tabular}


Table 10 Assessment of a particular work situation

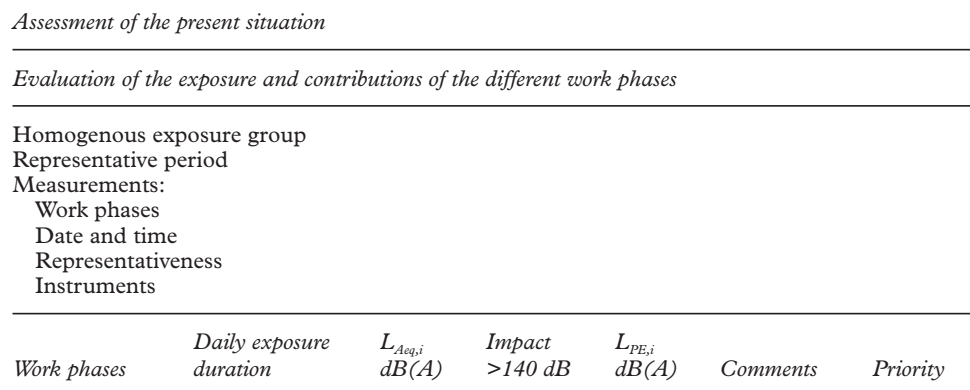

\begin{tabular}{ll}
\hline & \multicolumn{1}{c}{$L_{P E} d B(A)=$} \\
\hline Interpretation: & $\begin{array}{l}\text { Discomfort } \\
\text { Risk of hearing impairment }>25 \mathrm{~dB}: \\
\text { Risk of hearing impairment }>50 \mathrm{~dB}:\end{array}$ \\
\hline
\end{tabular}

- Place heavy gaskets

- Use heavier barriers

- Reorganisation of the work

- Reorganisation of the work sites, the work sequences and the work duration to reduce the duration of the exposure to the highest levels of noise.

(3) Report the actions envisaged as in table 11 , specifying who will do what and when.

(4) Exposure of the workers: future situation

Table 11 Actions resulting from table 10

\begin{tabular}{llllll}
\hline & Actions & & \\
\cline { 2 - 4 } Items & What? & Who? & When? & Priority \\
\hline Work techniques & & & \\
Workplaces & & & \\
Noise sources & & & \\
Noise propagation & & & \\
Room acoustics & & & \\
Room insulation & & & \\
Work organisation & & & \\
Work duration & & & \\
\hline
\end{tabular}

Table 12 Future actions

Future situation

Evaluation of the exposure and contributions of the different work phases

\begin{tabular}{lllllll}
\hline & Daily duratio & $L_{A e q, i}$ & Impact & $L_{P E, i}$ & & \\
Work phases & $n$ of exposure & $d B(A)$ & $>140 d B$ & $d B(A)$ & Comments & Priority
\end{tabular}

$$
L_{P E} d B(A)=
$$

Maximum admissible level of noise exposure

Interpretation residual risk:

Discomfort:

Risk of hearing impairment $>25 \mathrm{~dB}$ :

Risk of hearing impairment $>50 \mathrm{~dB}$ :

\begin{tabular}{ll}
\hline \multicolumn{1}{c}{ Need for a stage 3, Expertise } \\
\hline Personal protection & $\begin{array}{l}\text { What ? } \\
\text { By whom ? } \\
\text { When? }\end{array}$ \\
Medical surveillance (audiometric examinations)
\end{tabular}

- Estimate the exposure of the workers in the future situation

- Use the procedure described in section 2.1, taking into consideration

- Prevention and control measures at the source listed above

- Modifications for the room and partitions

- Reorganisation of work.

- Compute the personal noise exposure level $\mathrm{L}_{\mathrm{PE}}$.

- Estimate the residual risk

- Residual type of risk: discomfort, or hearing impairment

- Prediction of the risk of hearing impairment

- Estimate the need for a stage 3, expertise, on the basis of the residual risk: what priority and what objectives?

- Decide what short term protective measures must be taken: personal protection: what protection, carried out by whom, when, and for how long?

- Organise the medical surveillance: programme of audiometric examinations in the context of a hearing conservation programme

- Report this information in table 12.

STAGE 3, EXPERTISE

Objectives

- Through special measurements, better characterise some noises or certain acoustic phenomena in the work environment

- Through a finer analysis of activities and noise conditions, identify ultimate prevention or control modifications.

Who?

People of the company with the assistance of experts possessing:

- The specialised measuring equipment (frequency analysers, reverberation time measuring systems) and the training to use these techniques appropriately and interpret the results

- The technical expertise for the identification of particular technical solutions.

How?

As this stage concerns special acoustic conditions, it is neither necessary nor possible to define a systematic procedure. The expert should be able to define the measuring and investigation techniques most suitable for the problem in hand.

The expertise must obviously include the justification of techniques used.

At the end, the different steps of stage 2, analysis, must be repeated, and in particular

- The prevention or control measures recommended

- Who makes what and when?

- The residual risk after prevention or control

- The personal protection aspects

- The medical surveillance

This synthesis must again be made by the people of the company themselves with the assistance of specialists and experts. 
1 Pell S. An evaluation of a hearing conservation program. $A m$ Ind Hyg Assoc $\mathcal{F}$ 1972;33:60-70.

2 Hager WI, Hoyle ER, Hermann ER Efficacy of enforcement in an industrial hearing conservation program. Am Ind Hyg Assoc f 1982;43:455-65.

3 Melnick W. Evaluation of industrial hearing conservation programs: a review and analysis. Am Ind Hyg Assoc $\mathcal{F} 1984$ 45:459-67.

4 Royster LH, Royster JD. Education and motivation. In: Berger EH, Ward WD, Morrill JC, et al, eds. Noise and hearing conservation manual. Fairfax, Virginia, USA: American Industrial Hygiene Association, 1986:383-416.

5 Harrison RK. Hearing conservation: implementing and evaluating a program. AAOHN fournal 1989;37:107-111.

6 Reynolds JL, Royster LH, Pearson RG. Hearing conservation programs (HCPs): the effectiveness of one company's HCP in a 12 h work shift environment. Am Ind Hyg Assoc 1990;51:437-46.

7 Starck J. Noise and vibration. In: Brune D, Gerhardsson G, Crockford GW, et al, eds. The workplace. Vol 1. Fundamentals of health, safety and welfare. Geneva: International tals of health, safety and welfare. Geneva: International Labour Office, International Occupation
Health Information Centre (CIS), 1997.

8 Royster JD, Royster LH. Hearing conservation programs: practical guidelines for success. Chelsea, Michigan, USA: Lewis, 1990.

9 Suter AH, Franks R. A practical guide to effective hearing conservation programs in the workplace. Cincinnati, Ohio, USA National Institute for Occupational Safety and Health Department of Health and Human Services 1990. (Pub No $90-120$.

10 Leinster R, Baum J, Rong D, et al. Management and motivational factors in the control of noise-induced hearing-loss. Ann Occup Hyg 1994;38:649-62.
11 Mital A, Ayoub M, Kumar S, et al, eds. Industrial and occupational ergonomics: user's encyclopedia. International fournal of Industrial Engineering: Theory, Applications and Practice 1998.

12 Malchaire J, Piette A. A comprehensive strategy for the assessment of noise exposure and risk of hearing impairment. Ann Occup Hyg 1997;41:467-84.

13 Malchaire J, Gebhardt HJ, Piette A. Strategy for evaluation and prevention of risk due to work in thermal environment. Ann Occup Hyg 1999;43,5:367-76.

14 European Union. Council Directive on the protection of workers from the risks related to exposure to noise at work (86/188/EEC). Official Fournal of the European Communities 1986. (No L 137/28-No L 137/34.)

15 International Standards Organisation 9921-1. Ergonomic assessment of speech communication. Part 1: Speech interference level and communication distances for persons with normal hearing capacity in direct communication (SIL method). Geneva: ISO, 1996.

16 International Standards Organisation 1999. Acoustics: determination of occupational noise exposure and estimation of noise induced hearing impairment. Geneva: ISO, 1992.

17 Franks JR, Stephenson MR, Merry CJ, eds. Preventing occupational hearing loss: a practical guide. Cincinnati: National Institute for Occupational Safety and Health, 1996:92.

18 Berger EH, Franks JR, Lindgren F. International review of field studies of hearing protector attenuation. In: Axelsson A, Brochgrevink H, Hamernik RP, et al, eds. Scientific basis of noise-induced hearing loss. New York: Thieme, 1996:361- 